Bangladesh J. Plant Taxon. 27(2): 359-375, 2020 (December)

(C) 2020 Bangladesh Association of Plant Taxonomists

\title{
LEAFY VEGETABLES IN CHAPAI NAWABGANJ DISTRICT OF BANGLADESH FOCUSING ON MEDICINAL VALUE
}

\author{
A.H.M. Mahbubur Rahman and Most. Mejanara Khatun \\ Plant Taxonomy Laboratory, Department of Botany, Faculty of Biological Sciences, \\ University of Rajshahi, Rajshahi-6205, Bangladesh
}

Keywords: Assessment; Leafy vegetable taxa; Indigenous uses; Chapai Nawabganj District; Bangladesh.

\begin{abstract}
Leafy vegetables in Chapai Nawabganj district of Bangladesh was studied from January to December 2019. A total of 111 species belonging to 59 genera under 30 families were recorded, out of which, $52.25 \%$ species were wild and $46.84 \%$ species were cultivated in this study. Status of occurrence has been recorded for proper conservation management and sustainable utilization of the taxa resulting in $81.98 \%$ to be common, $17.11 \%$ as rare and $0.90 \%$ are found as vulnerable in the study area. A total of 93 medicinal plants have been documented with their uses for the cure of more than 53 diseases. The study showed that the people of Chapai Nawabganj district use leafy vegetables to treat their diseases. Therefore, the documented leafy vegetables should be further investigated for their efficacy and safety to be integrated into conventional medicine. Further more these leafy vegetables need to be conserved for their sustainable utilization.
\end{abstract}

\section{Introduction}

Leaf vegetables, also called potherbs, greens, or leafy greens, are plant leaves eaten as a vegetable, sometimes accompanied by tender petioles and shoots. Nearly one thousand species of plants with edible leaves are known (Rashid, 1999). Leaf vegetables most often come from short-lived herbaceous plants, such as lettuce and spinach. Woody plants of various species (Moringa oleifera, Murraya koinigii, Morus alba, Toona cilliata etc.) also provide edible leaves (NRC, 2015). They constitute a major portion of our diet and play an important part in alleviating malnutrition. FAO (2012) has estimated that about 870 million people are chronically undernourished in the period 2010-12 representing $12.5 \%$ of the global population, or one in eight people. In order to arrest the undernourished situation, much attention has been paid on the exploitation and utilization of unusual plant materials for food (Kawatra et al., 2001). Indigenous (traditional) vegetables are best defined as species that are locally important for the sustainability of economics, human nutrition and health, and social systems. Over the last decade, many studies have shown that fresh vegetables constitute important functional food components by contributing vitamins, iron, folic acid, mineral, biologically active compounds and photosysthetic pigments (Kmiecik et al., 2001; Su et al., 2002; Kimura and Rodriguez-Amaya, 2003). Vegetables also contain antioxidants which offer protection against many chronic disease including heart disease and certain types of cancer (Saxena, 1999).

In Bangladesh, people have a long heritage of taking leafy vegetables. However, very little attempt has been made to study the leafy vegetables of Bangladesh although they constitute a large proportion of the daily diet of the rural dweller of the country (Ali et al., 1977; Sarker and

${ }^{1}$ Corresponding author, E-mail: drrahmanahmm@ ru.ac.bd 
Hossain, 2009; Hassan, 2010, Rahman et al., 2015; Khatun et al., 2013; Rashid 1999). Despite the importance of leafy vegetables in the present day human lives, no systematic work has been carried out in the study area to identify and document the plant species. In view of potential beneficial attributes of leafy vegetables, there is a need to explore, identify and document the leafy vegetables of Chapai Nawabganj district, Bangladesh.

\section{Materials and methods}

\section{Study area}

Chapai Nawabganj is located on the north-western part of Bangladesh. It is a part of the Rajshahi Division and known for its special tone of local dialect. The north and west part of Chapai Nawabganj is bounded by Malda and Murshidabad district of India, east is by Naogaon District, and south-east is by Rajshahi District (BPC, 2001).

\section{Data collection}

The work is based on fresh materials collected during twenty three visits to Chapai Nawabganj distict, Bangladesh from January to December 2019 to cover the seasonal variations as well. The visits covered all types of habitats, particular river bank, slope, village grove, fruit gardens and roadsides of the study area. Medicinal information was obtained through semistructured interviews with knowledgeable people such as local 'Kabiraj'(local herbal doctor) and elderly people. A total of 161 informants having an age rangeing from 21 to 69 years were interviewed using semi-structured interviewed method (Alexiades, 1996). Plant parts with either flower of fruits collected using traditional herbarium techniques to make voucher specimens for documentation.

\section{Plant identification}

Collected specimens have been critically examined, studied and identified. Identifications have been confirmed by consulting standard literatures (Hooker, 1877; Prain, 1903) and Herbarium of Rajshahi University (HRU). Nomenclature has been updated following recent literature (Ahmed et al., 2008-2009; Huq, 1986, and Pasha and Uddin, 2013).

\section{Results and Discussion}

Documentation of leafy vegetable taxa in Chapai Nawabganj district of Bangladesh was investigated. Out of 111 species, 106 belong to angiosperms, and 5 to pteridophytes. Among the angiospermic taxa Magnoliopsida is represented by 96 taxa and Liliopsida is represented by 10 taxa (Table 1). Distribution of leafy vegetables in the families shows variation. Cucurbitaceae is the dominant family represented by 16 species, followed by Amaranthaceae (13 species), Brassicaceae (9 species), Fabaceae (8 species), Araceae (8 species), Solanaceae (7 species), Convolvulaceae (7 species), Malvaceae (4 species) and Polygonaceae (4 species) (Table 1; Fig. 3). A single species is represented by 9 families while 2 to 3 species is represented by 12 families. Out of recorded species, $52.25 \%$ species were wild and $46.84 \%$ species were cultivated in the study area (Fig. 2). Status of occurrence has been recorded for proper conservation management and sustainable utilization of the taxa resulting in $81.98 \%$ ) to be common, $17.11 \%$ as rare and $0.90 \%$ are found as vulnerable in the study area (Fig. 1).

The collected information is comparable with the result of other studies in Bangladesh and abroad. In Bangladesh, 186 species were recorded as leafy vegetables (Khatun et al, 2013). A total of 24 species belonging to 22 genera and 16 families were collected and identified in Bogra district (Rahman et al, 2015). Narayanan and Kumar (2007) in India recorded a total of 102 wild edible leaves in Paniya, Kattunaikka and Kuruma tribes, but families consume 88, 43 and 21, 
Table 1. Diversity of leafy vegetables in Chapai Nawabganj district, Bangladesh.

\begin{tabular}{|c|c|c|c|c|c|}
\hline Scientific name & Bangla name & Family & $\begin{array}{l}\text { Status of } \\
\text { occurrence }\end{array}$ & $\begin{array}{l}\text { Flowering } \\
\text { time }\end{array}$ & $\begin{array}{l}\text { Voucher } \\
\text { number }\end{array}$ \\
\hline Acalypha indica $\mathrm{L}$. & Muktajhuri & Euphorbiaceae & Common & Mar-Oct & MK 91 \\
\hline Amaranthus blitum L. & Notiya shak & Amaranthaceae & Common & Jan-Dec & MK 29 \\
\hline Amaranthus gangeticus L. & Lal shak & Amaranthaceae & Common & Jun-Aug & MK 124 \\
\hline Amaranthus lividus Roxb. & Gobranotey & Amaranthaceae & Common & Jan-Dec & MK 31 \\
\hline Amaranthus oleracea L. & Data shak & Amaranthaceae & Common & Jan-Dec & MK 122 \\
\hline Amaranthus polygonoides $\mathrm{L}$. & Notey shak & Amaranthaceae & Common & Jan-Dec & MK 33 \\
\hline Amaranthus spinosus L. & Katanotey & Amaranthaceae & Common & Jan-Dec & MK 120 \\
\hline Amaranthus tricolor L. & Lal shak & Amaranthaceae & Common & Jan-Dec & MK 35 \\
\hline Amaranthus viridis L. & Notey shak & Amaranthaceae & Common & Jan-Dec & MK 118 \\
\hline Alternanthera sessilis (L.) R. Br. & Sachishak & Amaranthaceae & Common & May- Oct & MK 37 \\
\hline $\begin{array}{l}\text { Alternanthera philoxeroides } \\
\text { (Mart.) Griseb. }\end{array}$ & Malancha & Amaranthaceae & Common & Mar-Jun & MK 116 \\
\hline Alternanthera bettzickiana $\mathrm{L}$. & Malancha & Amaranthaceae & Rare & Mar-Jun & MK 39 \\
\hline Azadirachta indica A. Juss. & Nimpata & Meliaceae & Common & Mar-Apr & MK 93 \\
\hline Alocasia indica Schott. & Mankochu & Araceae & Common & Aug-Oct & MK 117 \\
\hline $\begin{array}{l}\text { Amorphophalus bulbifer (Roxb.) } \\
\text { Blume }\end{array}$ & Olkochu pata & Araceae & Common & Jul- Aug & MK 36 \\
\hline Allium cepa $\mathrm{L}$. & Piaj. & Liliaceae & Common & Feb-Apr & MK 125 \\
\hline Allium sativum $\mathrm{L}$. & Rasun. & Liliaceae & Common & Feb-Apr & MK 28 \\
\hline $\begin{array}{l}\text { Angiopteris evecta (Forst.) } \\
\text { Hoffm. }\end{array}$ & Dhekishak & Angiopteridaceae & Rare & Jan-Dec & MK 127 \\
\hline Argemone mexicana $\mathrm{L}$. & Sialkata & Papaveraceae & Common & Jan-Dec & MK 133 \\
\hline Basella alba $\mathrm{L}$. & Puishak & Basellaceae & Common & Nov-Feb & MK 110 \\
\hline $\begin{array}{l}\text { Benincasa hispida (Thunb.) } \\
\text { Cogn. }\end{array}$ & Chalkumra. & Cucurbitaceae & Common & May-Sep & MK 57 \\
\hline Boerhaavia repens $\mathrm{L}$. & Punarnava & Nyctaginaceae & Common & Jan-Dec & MK 130 \\
\hline Brassica alba Hook. & $\begin{array}{l}\text { Sada sorisha } \\
\text { shak }\end{array}$ & Brassicaceae & Common & Mar- May & MK 80 \\
\hline Brassica campestris Roxb. & Sorisha shak & Brassicaceae & Common & Mar- May & MK 75 \\
\hline Brassica juncea $\mathrm{L}$. & Rai sorisha & Brassicaceae & Common & Mar- May & MK 78 \\
\hline Brassica napus L. & Kalo Sarisha & Brassicaceae & Common & Mar- May & MK 77 \\
\hline $\begin{array}{l}\text { Brassica oleracea L. var. } \\
\text { botrydis }\end{array}$ & Fulkopi & Brassicaceae & Common & Dec-Feb & MK 76 \\
\hline $\begin{array}{l}\text { Brassica oleracea L. var. } \\
\text { capitata }\end{array}$ & Badhakopi & Brassicaceae & Common & Dec-Feb & MK 79 \\
\hline $\begin{array}{l}\text { Brassica oleracea L. var. } \\
\text { gangyloides }\end{array}$ & Olkopi & Brassicaceae & Common & Dec-Feb & MK 74 \\
\hline
\end{tabular}


Table 1 contd.

\begin{tabular}{|c|c|c|c|c|c|}
\hline Scientific name & Bangla name & Family & $\begin{array}{l}\text { Status of } \\
\text { occurrence }\end{array}$ & $\begin{array}{l}\text { Flowering } \\
\text { time }\end{array}$ & $\begin{array}{l}\text { Voucher } \\
\text { number }\end{array}$ \\
\hline Brassica rapa $\mathrm{L}$. & Shalgam & Brassicaceae & Common & Jan-Mar & MK 81 \\
\hline Bacopa monnieri (L.) Pennel. & Brahmishak & Scrophulariaceae & Rare & Jun-Aug & MK 42 \\
\hline Chenopodium album $\mathrm{L}$. & Bathua Shak & Chenopodiaceae & Common & Dec-Feb & MK 128 \\
\hline Chenopodium ambrosoides $\mathrm{L}$. & Bonbothua & Chenopodiaceae & Common & Mar- May & MK 27 \\
\hline Celosia cristata $\mathrm{L}$. & Moragphul & Amaranthaceae & Common & Jan-Dec & MK 114 \\
\hline Centella asiatica (L.) Urb. & Thankuni & Apiaceae & Common & Feb-May & MK 58 \\
\hline Coriandrum sativum $\mathrm{L}$. & Dhaniya. & Apiaceae & Common & Dec-Feb & MK 97 \\
\hline Capsicum frutescens $\mathrm{L}$. & Marich & Solanaceae & Common & Jan-Dec & MK 56 \\
\hline Cicer arietinum $\mathrm{L}$. & Boot & Fabaceae & Common & Jan-Dec & MK 70 \\
\hline Corchorus capsularis L. & Deshipat & Tiliaceae & Common & Mar-Jun & MK 102 \\
\hline Corchorus olitorius L. & Pat shak & Malvaceae & Common & Mar-Jun & MK 98 \\
\hline Coccinia cordifolia (L.) Cogn. & Telakucha & Cucurbitaceae & Common & Mar-Dec & MK 96 \\
\hline Coccinia grandis (L.) Voigt. & Telakucha & Cucurbitaceae & Common & Mar- Dec & MK 59 \\
\hline Cucumis melo L. & Bangi & Cucurbitaceae & Common & Jan-May & MK 94 \\
\hline Cucumis sativus $\mathrm{L}$. & Shosha & Cucurbitaceae & Common & Jan- Dec & MK 61 \\
\hline Cucurbita maxima Duch. & Mistikumra & Cucurbitaceae & Common & Mar- Aug & MK 92 \\
\hline Cucurbita sativus Duch. & Kumra & Cucurbitaceae & Rare & Jan- Dec & MK 63 \\
\hline Cucurbita pepo DC. & Mistikadu & Cucurbitaceae & Common & Mar-Aug & MK 90 \\
\hline Cucurbita moschata Duch. & Mistikadu & Cucurbitaceae & Common & Mar- Aug & MK 65 \\
\hline Colocasia esculenta (L.) Schott & Kachu & Araceae & Common & Dec-Mar & MK 119 \\
\hline Diplazium esculentum Retz. & Dhekishak & Athyriaceae & Frequent & Jan-Dec & MK 26 \\
\hline Diplazium polypodioides $\mathrm{Bl}$. & Dhekishak & Athyriaceae & Frequent & Jan-Dec & MK 129 \\
\hline Digera muricata (L.) Mart. & Boutibon shak & Amaranthaceae & Vulnerable & Feb-Jul & MK 41 \\
\hline Enhydra fluctuans Lour. & Helencha & Asteraceae & Common & Nov-Feb & MK 115 \\
\hline $\begin{array}{l}\text { Glinus oppositifolius (L.) Aug. } \\
\text { DC. }\end{array}$ & Gima-sak & Molluginaceae & Common & Jan-Dec & MK 45 \\
\hline Hibiscus cannabinus L. & Mestapat & Malvaceae & Common & Apr-Aug & MK 53 \\
\hline Hibiscus sabdariffa $\mathrm{L}$. & Lalmesta & Malvaceae & Common & Apr-Aug & MK 100 \\
\hline $\begin{array}{l}\text { Hygrophila auriculata (Schum.) } \\
\text { Heyne. }\end{array}$ & Puninnya shak & Acanthaceae & Rare & Jan-Dec & MK 113 \\
\hline Ipomoea aquatica Forssk. & Kalmi Shak & Convolvulaceae & Common & Jan-Oct & MK 105 \\
\hline Ipomoea batatas (L.) Lamk. & Misti Alu. & Convolvulaceae & Common & Jan-Dec & MK 48 \\
\hline Ipomoea cairica $($ L.) Sweet & Rail Lata & Convolvulaceae & Common & Jan-Dec & MK 107 \\
\hline $\begin{array}{l}\text { Ipomoea fistulosa (Mart. ex } \\
\text { Choisy) }\end{array}$ & Dholkolmi & Convolvulaceae & Common & Jan-Dec & MK 46 \\
\hline
\end{tabular}


Table 1 contd.

\begin{tabular}{|c|c|c|c|c|c|}
\hline Scientific name & Bangla name & Family & $\begin{array}{l}\text { Status of } \\
\text { occurrence }\end{array}$ & $\begin{array}{l}\text { Flowering } \\
\text { time }\end{array}$ & $\begin{array}{l}\text { Voucher } \\
\text { number }\end{array}$ \\
\hline Ipomoea indica (Burm.) Merr. & Kolmi & Convolvulaceae & Rare & Jan-Dec & MK 109 \\
\hline Ipomoea purpurea (L.) Roth. & Beguni Kolmi & Convolvulaceae & Rare & Jul- Sep & MK 44 \\
\hline Ipomoea quamoclit L. & Torulata & Convolvulaceae & Common & Jul-Sep & MK 111 \\
\hline Lasia spinosa (L.) Thw. & Kantakachu & Araceae & Rare & Jan-Dec & MK 34 \\
\hline Lactuca sativa $\mathrm{L}$. & Lettuce & Asteraceae & Common & Jan-Mar & MK 38 \\
\hline Lathyrus sativus L. & Kheshari & Fabaceae & Common & Jan-Mar & MK 23 \\
\hline Lagenaria siceraria (Mol.) Stan. & Lau & Cucurbitaceae & Common & Feb - Apr & MK 88 \\
\hline Luffa cylindrica (L.) Roem. & Dhundol pata & Cucurbitaceae & Common & Jun-Nov & MK 67 \\
\hline Lathyrus hirsutus L. & Bon kheshari & Fabaceae & Common & Jan- Mar & MK 85 \\
\hline Lathyrus sativus L. & Kheshari & Fabaceae & Common & Jan- Mar & MK 68 \\
\hline Lens culinaris Medik. & Musuri & Fabaceae & Common & Jan-Dec & MK 87 \\
\hline Moringa oleifera Lamk. & Sajna & Moringaceae & Common & Jan-Dec & MK 83 \\
\hline Momordica carantia $\mathrm{L}$. & Karolla & Cucurbitaceae & Common & May- Aug & MK 86 \\
\hline Momordica dioica Roxb. & Kakrol & Cucurbitaceae & Common & Jul-Dec & MK 69 \\
\hline Momordica cochichinensis Roxb. & Kakrol & Cucurbitaceae & Common & Mar- Sep & MK 84 \\
\hline Marsilea minuta (L.)Mant. & Susni shak & Marsileaceae & Common & Jan-Dec & MK 24 \\
\hline Marsilea quadrifolia $\mathrm{L}$. & Susni shak & Marsileaceae & Common & Jan-Dec & MK 131 \\
\hline Malva verticilata $\mathrm{L}$. & Napashak & Malvaceae & Rare & Jul-Sep & MK 55 \\
\hline Mollugo pentaphylla L. & Tita shak & Molluginaceae & Rare & Jan-Dec & MK 108 \\
\hline Mollugo spergula $\mathrm{L}$. & Gima shak & Molluginaceae & Rare & Jan-Dec & MK 47 \\
\hline Oxalis europea $\mathrm{L}$. & Amrul & Oxalidaceae & Common & Sep-Mar & MK 60 \\
\hline Oxalis corniculata $\mathrm{L}$. & Amrul & Oxalidaceae & Common & Sep-Mar & MK 95 \\
\hline Portulaca oleracea L. & Baranunia & Portulacaceae & Common & May- Jul & MK 112 \\
\hline Portulaca quadrifida L. & Chotonunia & Portulacaceae & Common & Jan-Dec & MK 43 \\
\hline Pisum sativum $\mathrm{L}$. & Matar. & Fabaceae & Common & Jan-Dec & MK 64 \\
\hline Phyllanthus niruri L. & Vuiamla & Euphorbiaceae & Common & Apr-Sep & MK 62 \\
\hline Paederia foetida $\mathrm{L}$. & Gandhabhaduli & Rubiaceae & Common & Jan-Dec & MK 40 \\
\hline Raphanus sativus L. & Mula & Brassicaceae & Common & Jan-Mar & MK 72 \\
\hline Rumex vesicarius $\mathrm{L}$. & Takpalong & Polygonaceae & Rare & Jul-Aug & MK 106 \\
\hline Rumex dentatus L. & Bon Palong & Polygonaceae & Common & Jul-Aug & MK 49 \\
\hline Rumex maritimus L. & Bon Palong & Polygonaceae & Common & Jul-Aug & MK 104 \\
\hline Rumex sanguineus L. & Bon Palong & Polygonaceae & Common & Jul-Aug & MK 51 \\
\hline Sesuvium portulacastrum L. & Nuna shak & Aizoaceae & Common & Jan-Dec & MK 25 \\
\hline Solanum americanum L. & Tit Begun & Solanaceae & Common & Jan-Dec & MK 99 \\
\hline
\end{tabular}


Table 1 contd.

\begin{tabular}{|c|c|c|c|c|c|}
\hline Scientific name & Bangla name & Family & $\begin{array}{l}\text { Status of } \\
\text { occurrence }\end{array}$ & $\begin{array}{l}\text { Flowering } \\
\text { time }\end{array}$ & $\begin{array}{l}\text { Voucher } \\
\text { number }\end{array}$ \\
\hline Solanum nigrum $\mathrm{L}$. & Tit Begun & Solanaceae & Common & Jan-Dec & MK 54 \\
\hline Solanum indicum $\mathrm{L}$. & Tit Begun & Solanaceae & Rare & Jan-Dec & MK 101 \\
\hline Solanum tuberosum $\mathrm{L}$. & Alu & Solanaceae & Common & Oct-Feb & MK 52 \\
\hline Solanum villosum $\mathrm{L}$. & Titbegun & Solanaceae & Common & Jan-Dec & MK 103 \\
\hline Solanum filisifolium L. & Titbegun & Solanaceae & Common & Jan-Dec & MK 50 \\
\hline Spinacea oleracea L. & Palong Shak & Chenopodiaceae & Common & Nov-Feb & MK 126 \\
\hline Trichosanthes anguina $\mathrm{L}$. & Chichinga & Cucurbitaceae & Common & Nov-Apr & MK 71 \\
\hline Trichosanthes bracteata Lam. & Makal. & Cucurbitaceae & Rare & Jul-Dec & MK 82 \\
\hline Trichosanthes dioica Roxb. & Potol. & Cucurbitaceae & Common & Apr- Aug & MK 73 \\
\hline $\begin{array}{l}\text { Thyphonium trilobatum (L.) } \\
\text { Schott. }\end{array}$ & Ghet Kochu & Araceae & Rare & Jan-Dec & MK 30 \\
\hline Vigna mungo (L.) Hepper & Mashkalai & Fabaceae & Common & Nov-Jan & MK 66 \\
\hline Vigna sinensis (L.) Endl. & Borboti & Fabaceae & Rare & Apr-Jul & MK 89 \\
\hline Xanthosoma atrovirens $\mathrm{L}$. & Moulovikochu & Araceae & Rare & Aug-Oct & MK 121 \\
\hline Xanthosoma sagittifolium $\mathrm{L}$. & Mukhikachu & Araceae & Rare & Aug-Oct & MK 32 \\
\hline Xanthosoma violaceum $\mathrm{L}$. & Dudh kachu & Araceae & Rare & Aug-Oct & MK 123 \\
\hline Xanthium strumarium L. & Ghagra & Asteraceae & Common & Jan-Dec & MK 136 \\
\hline
\end{tabular}

Jan = January, Feb = February, Mar = March, Apr = April, Ma = May, Jun = June, Jul $=$ July, Aug $=$ August, Sep $=$ September, Oct $=$ October, Nov $=$ November, Dec $=$ December, $\quad \mathrm{C}=$ Common, Vul $=$ Vulnerable, $\mathrm{R}=$ Rare.

respectively. In South Africa, Vorster et al. (2007) recorded the following species: Amaranthus spp.; Bidens pilosa; Chenopodium album; Corchorus spp.; Cucurbita spp.; Momordica balsamina; and Vigna unguiculata as some of the more popular leafy vegetables in areas where they are widespread. In East and West Usambaras, Tanzania, Vainio-Mattila (2000) documented Acanthaceae, Amaranthaceae, Asteraceae, and Brassicaceae as the most important families of wild green leafy vegetables, among 26 reported families. In Kenya and other parts of East Africa traditional leafy vegetables are used by both rural and urban communities and include several families such as Amaranthaceae, Basellaceae, Brassicaceae, Cucurbitaceae, and Tiliaceae (Abukutsa and Onyango, 2005). Further, in Andhra Pradesh, India; Reddy et al. (2007) report 69 families of wild food plants, where four families: Amaranthaceae (11 species); Rubiaceae (9 species); Euphorbiaceae (8 species) and Papilionaceae (7 species); have a high number of species, with Amaranthaceae family having the highest number of species. Fleuret (1979) recorded more than 15 species of wild leafy vegetables in her study in the Lushoto district, Tanzania. Woodcook's (1995) studied on indigenous knowledge and forest use in the East Usambaras in Tanzania documents 25 wild leafy vegetable species. So far the information available, no published data recorded on the leafy vegetables in Chapai Nawabganj district, Bangladesh. The present study will also help in identifying the important leafy vegetables for future reference.

Present study demonstrated that there is an urgent need for documentation of traditional knowledge related to the intangible cultural heritage concerning wild vegetables are utilized. The 
utilization and cultivation of these vegetables should be promoted to maintain the dietary needs of the household in Chapai Nawabganj district. The study can provide a baseline data that may be helpful for prioritization of conservation through sustainable use and management of the resources.

\section{Medicinally Important Leafy Vegetables}

Out of 111 species, 93 medicinal plants have been documented with their uses for the cure of more than 53 diseases. The medicinal plants are used by the local people to cure many the diseases, especially for anemia, asthma, burning sensation, blood diseases, bronchitis, cough, chicken pox, constipation, dysentery, diarrhea, diabetes, eczema, fever, headache, itches, jaundice, menstrual disease, paralysis, piles, sex problems, skin diseases, snake-bite, toothache, worm, wound etc. Different plant parts of different species are used as medicine for treating various diseases where; - leaf of $65.76 \%$, fruit of $17.11 \%$, root of $9.90 \%$, stem of $1.80 \%$, seed of $19.81 \%$, bulb of $1.80 \%$, Corm of $2.70 \%$, Flower of $2.70 \%$ and whole plant of $18.01 \%$ species were used as medicine (Fig. 4). The survey recorded 53 categories of uses of 93 medicinal leafy vegetables (Table 2). Out of 53 categories of ailments, Dysentery, fever, skin disease, cough, inflammation,

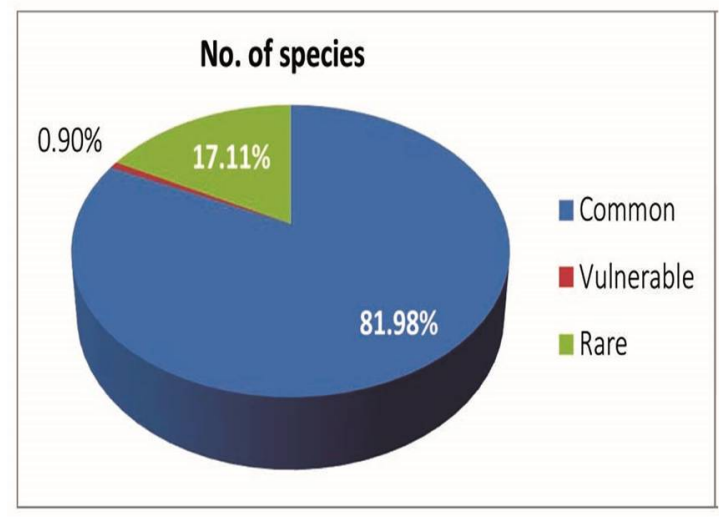

Fig. 1. Recorded status of occurrence in the study area.

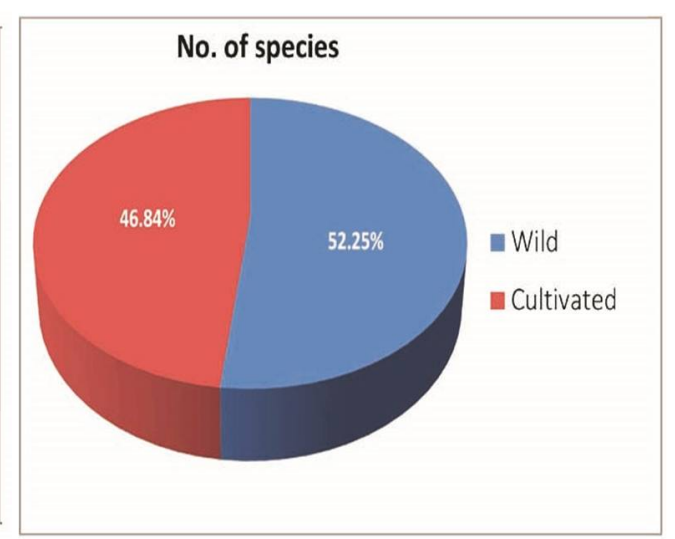

Fig. 2. Recorded wild and cultivated leafy vegetables in the study area

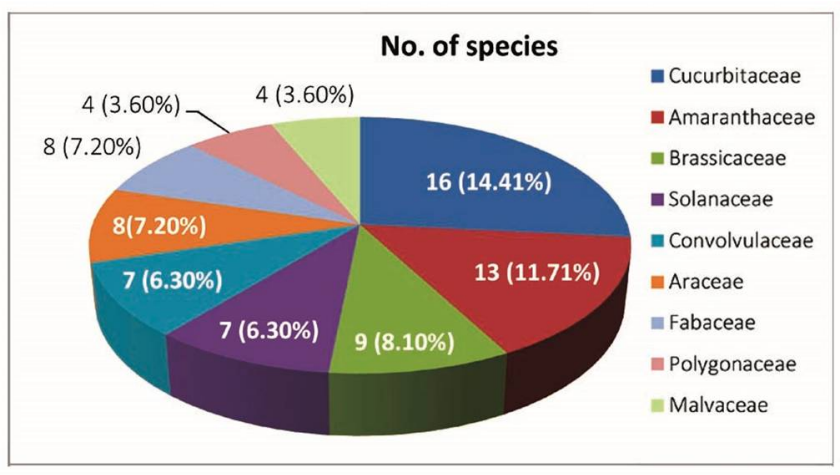

Fig. 3. Recorded dominant vegetable families in the study area 


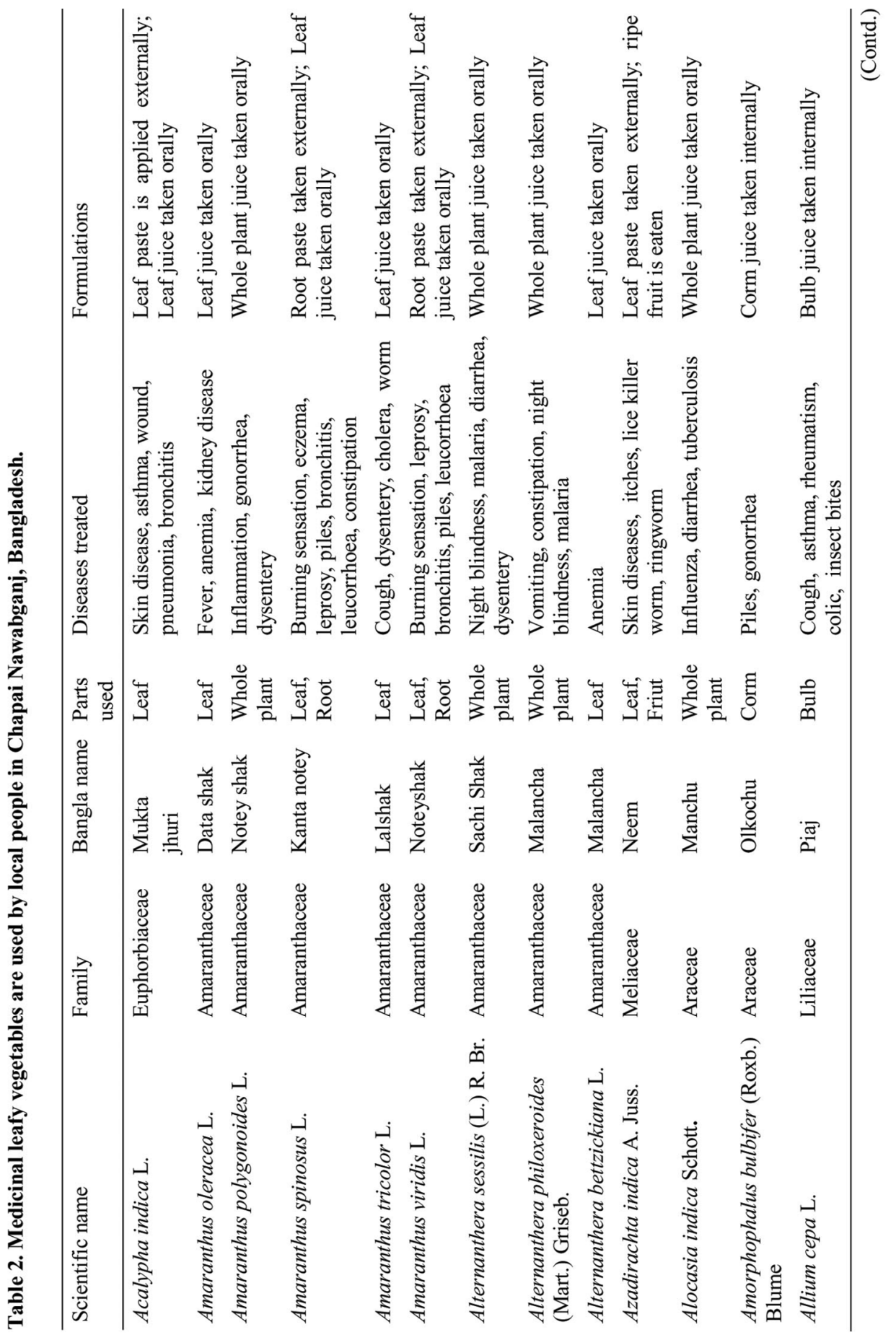




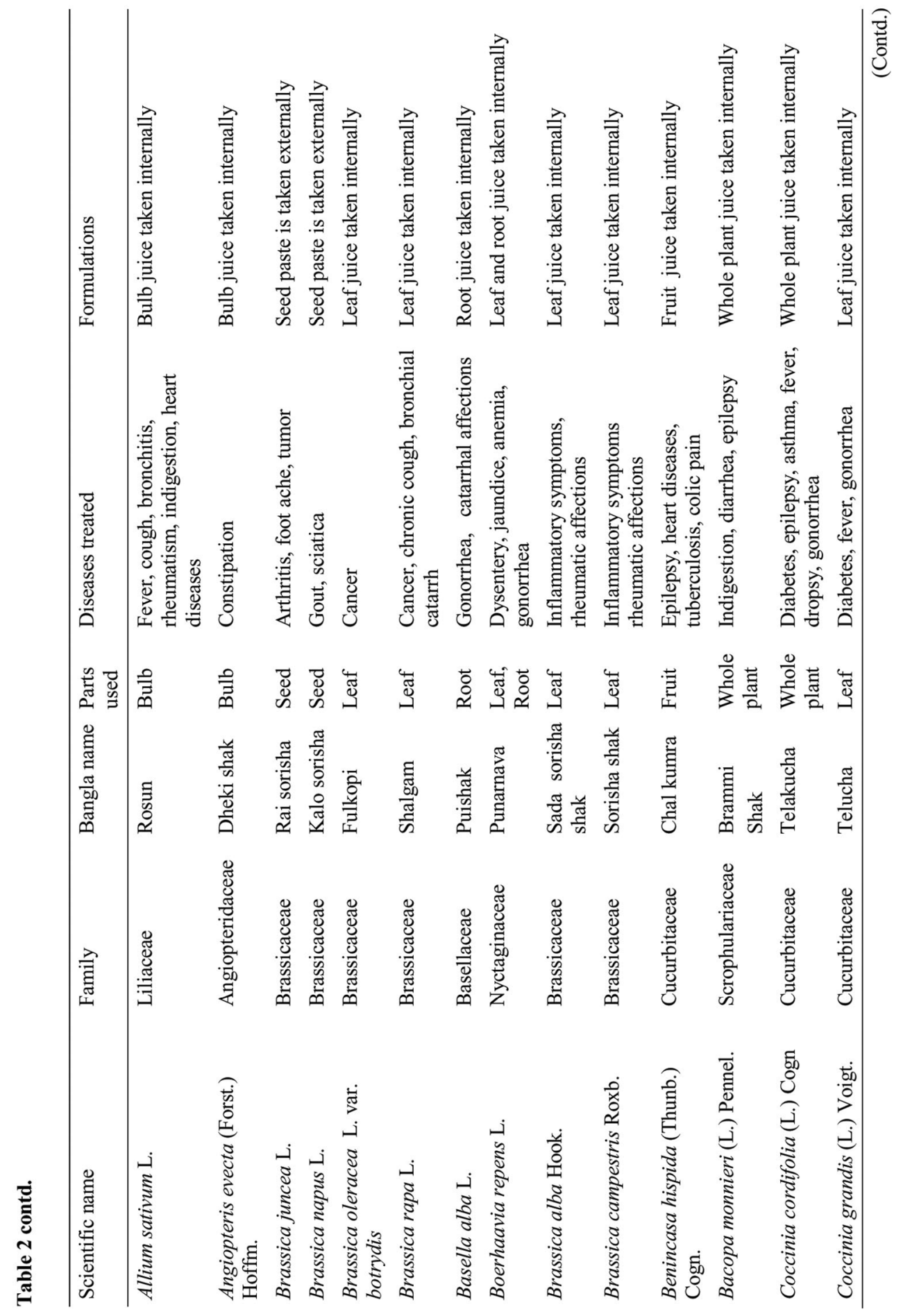




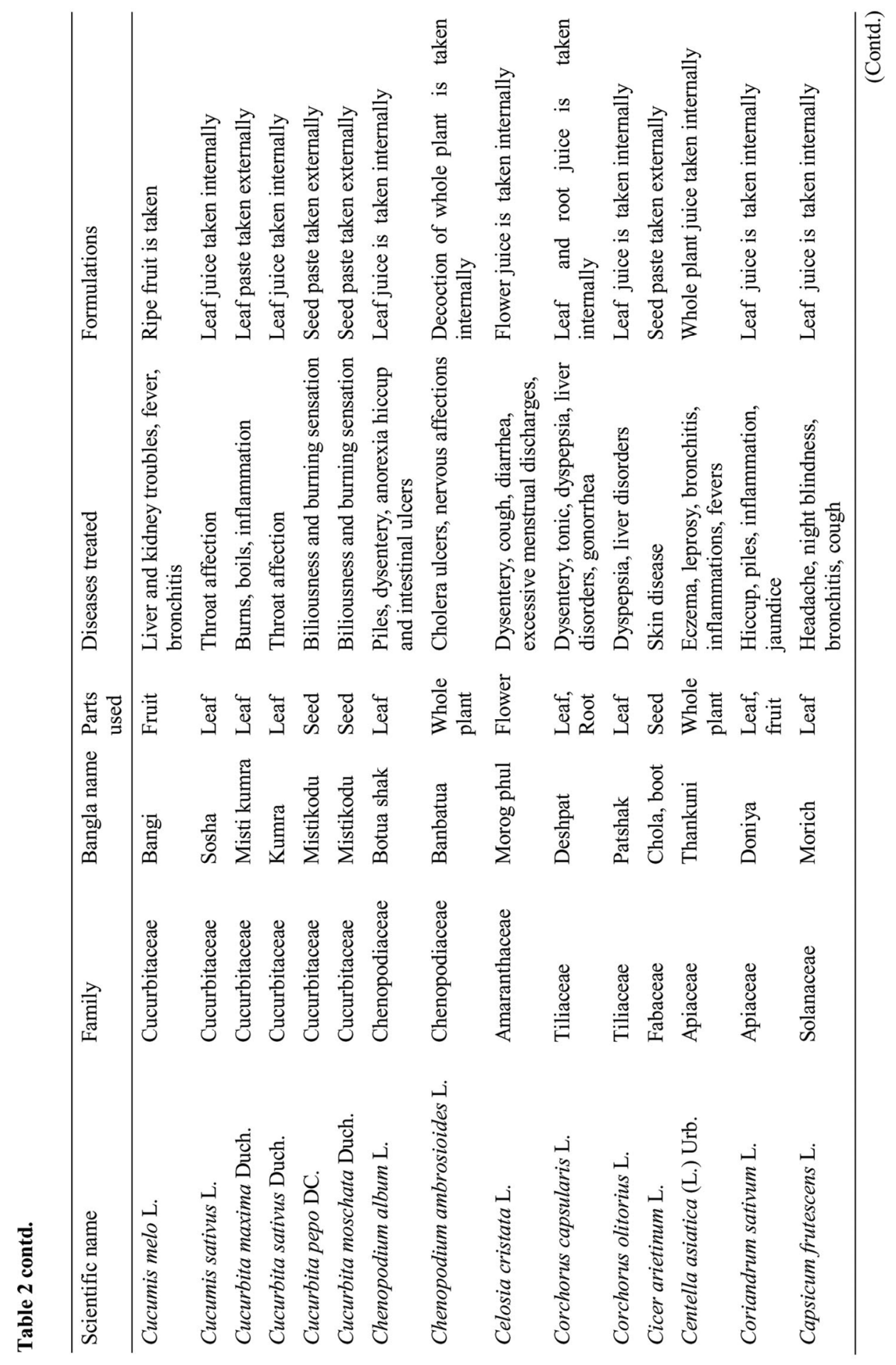




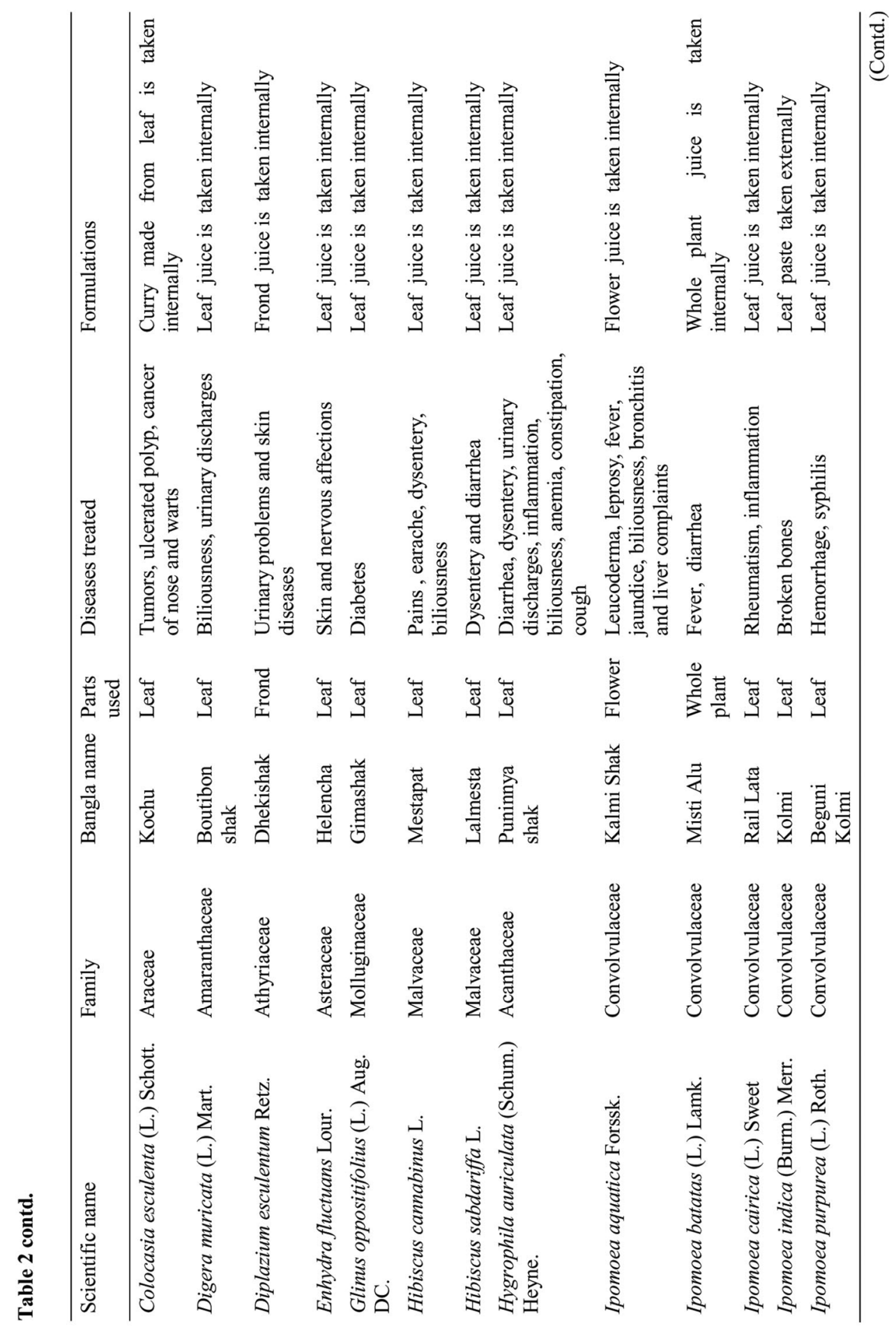




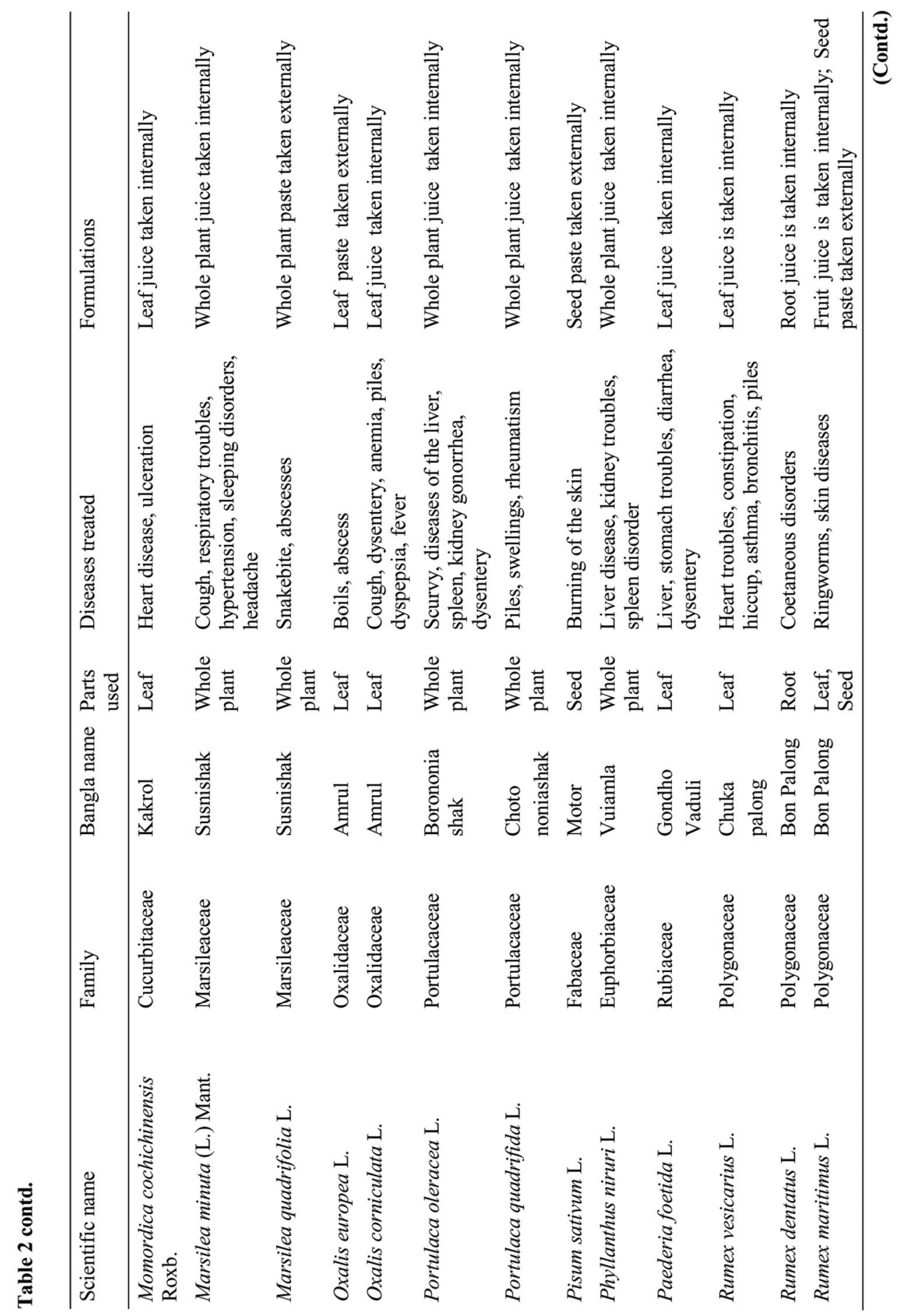




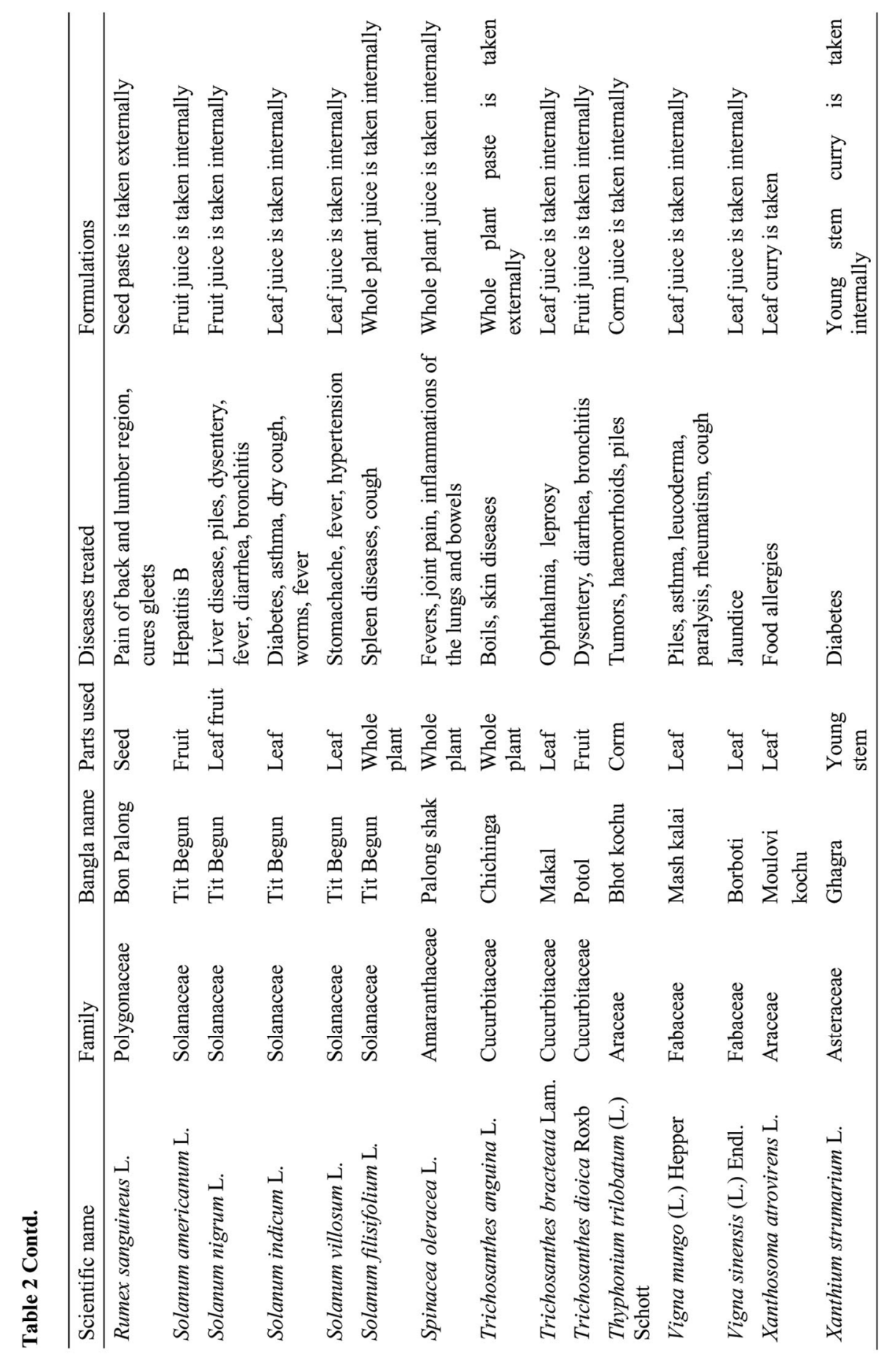




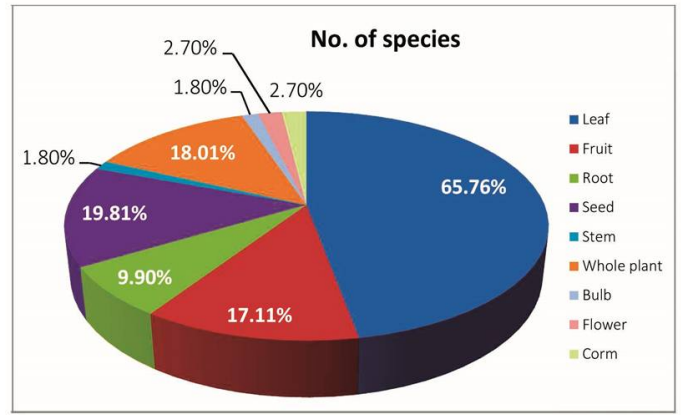

Fig. 4. Recorded plant parts used as medicine.

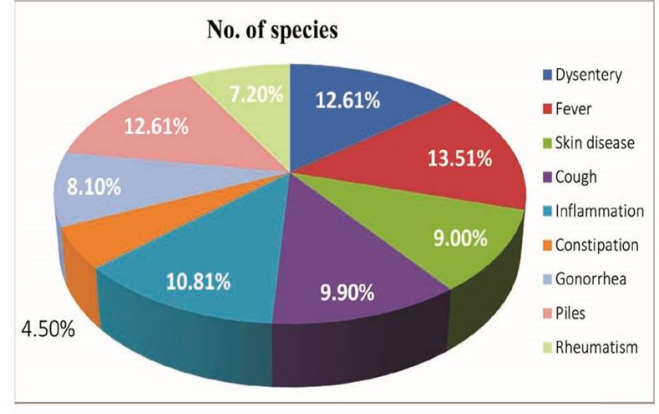

Fig. 5. Leafy vegetables used to treat different diseases recorded from the study.

constipation, gonorrhea, piles and rheumatism was dominant diseases in the study area (Fig. 5). This finding suggests that the leafy vegetables may possibly contain other phytochemical constituents which need to be investigated in future studies. This finding of common medicinal plant families in this study is in agreement with Anisuzzaman et al (2007); Ghani (2003); Khan (1998), Choudhury and Rahmatullah (2012), Faruque and Uddin (2014), Uddin and Hassan (2014), Uddin et al., (2015), and Yusuf et.al (2006). The present study revealed that medicinal plants still play an important in the primary health care of the rural communities. The information gathered from the local traditional healers are useful for further researchers in the field of ethnobotany, taxonomy and development of new drug from natural resources.

\section{Acknowledgements}

The authors are grateful to the local people in Chapai Nawabganj district of Bangladesh for their co-operation and help during the research work. The authors are also thanks to the Ministry of Science and Technology (MOST), Government of the People's Republic of Bangladesh for financial support to complete this research work.

\section{References}

Abukutsa, M.O. and Onyango, J.C. 2005. Conservation and seedproduction of African leafy vegetables at Maseno University botanic garden, Kenya. African Crop Science Conference Proceedings 7, 1201-1204.

Ahmed, Z.U., Hassan, M.A., Begum, Z.N.T., Khondker, M., Kabir, S.M.H., Ahmad, M., Ahmed, A.T.A., Rahman, A.K.A. and Haque, E.U. (Eds). 2008-2009. Encyclopedia of Flora and Fauna of Bangladesh, Vols. 6-10, Asiat. Soc. Bangladesh, Dhaka.

Alexiades, M.N. (Ed). 1996. Selected Guidelines for Ethno Botanical Research: A Field Manual. The New York Botanical Garden, New York. 305 pp.

Ali, S.M.K., Malek, M.A., Jahan, K. and Salamtullah, Q. (eds). 1977 (reprint 1992). Deshio Khadyo-drobber Pustiman (Nutritional value of local foods). Institute of Nutrition and Food Science, University of Dhaka, Dhaka.

Anisuzzaman, M., Rahman, A.H.M.M., Rashid, M.H., Naderuzzaman, A.T.M. and Islam, A.K.M.R. 2007. An Ethnobotanical Study of Madhupur, Tangail. Journal of Applied Sciences Research. 3(7): 519-530.

Bangladesh Population Census (BPC) 2001, Bangladesh Bureau of Statistics; Cultural survey report of Gobindhaganj Upazila 2007.

Choudhury, A.R. and Rahmatullah M. 2012. Ethnobotanical study of wound healing plants among the folk medicinal practioners several district in Bangladesh. American- Eurasian Journal of Sustainable Development. 6(4): 371-377. 
FAO. 2012. The State of Food Insecurity in the World 2012. Rome, Italy.

Faruque, M.O. and Uddin, S.B. 2014. Ethnomedicinal study of the Marma community of Bandarban district of Bangladesh. Academia Journal of Medicinal Plants. 2(2): 014- 025.

Fleuret, A. 1979. Methods for evaluation of the role of fruits and wild greens inShambaa diet: A case study. Medical Anthropology 3, 249-269.

Ghani, A. 2003.Medicinal Plants of Bangladesh. Asiatic Society of Bangladesh, Dhaka.

Hassan, M.A. 2010. Deshio Shak Shobjir Pusti Upadhan, Veshojgun o Patthaya Bichar. The Royal Publishers, pp. 1-127.

Hooker, J.D. 1877. The Flora of British India, Vols. 1-7. L. Reeve \& Co. Ltd. Kent, London.

Huq, A.M. 1986. Plant Names of Bangladesh. Bangladesh National Herbarium, BARC, Dhaka, Bangladesh.

Kawatra, A., Singh, G. and Sehgal, S. 2001. Nutrition composition of selected green leafy vegetables, hervs and carrots. Plant Foods for Human Nutrition 56: 359-365.

Khan, M.S. 1998. Prospects of Ethnobotany and Ethnobotanical Research in Bangladesh. In:R.L. Banik, M.K. Alam, S.J. Pei and A. Rastogi (eds.), Applied Ethnobotany, BFRI, Chittagong, Bangladesh. Pp. 24-27.

Khatun, M., Hassan, M.A., Islam, S.N. and Rahman, M.O. 2013. Taxonomy of the Leafy Vegetables in Bangladesh. Bangladesh J. Plant. Taxon. 20(1): 95-123.

Kimura, M. and Rodriguez-Amaya, D.B. 2003. Carotenoid composition of hydroponic leafy vegetables. J. Agric. \& Food Chem. 51: 2603-2607. Kmiecik, W., Lisiewska, Z. and Jaworska, G. 2001. Effect of storage conditions on the technological value of dill (Anethum graveolens L.). Folia Horticulturea 13: 33-43.

Kmiecik, W., Lisiewska, Z. and Jaworska, G. 2001. Effect of storage conditions on the technological value of dill (Anethum graveolens L.). Folia Horticulturea 13: 33-43.

Narayanan, M.K.R. and Kumar, N.A. 2007. Gendered knowledge and changingtrends in utilization of wild edible greens in Western Ghats, India. IndianJournal of Traditional Knowledge 6(1), 204-216.

National Research Council (U.S.). 2015. Board on Science and Technology for International Development, Lost Crops of Africa: Vegetables, pp. 6

Pasha, M.K. and Uddin, S.B. 2013. Dictionary of Plant Names of Bangladesh.JanokalyanProkashani. Chittagong. Bangladesh.

Prain, D. 1903. Bengal Plants, Vols. 1-2. Botanical Survey of India, Calcutta.

Rahman, A.H.M.M., Akter, S., Rani, R. and Islam, A.K.M.R. 2015. Taxonomic Study of Leafy Vegetables at Santahar Pouroshova of District Bogra, Bangladesh with Emphasis on Medicinal Plants. International Journal of Advanced Research. 3(5): 1019-1036.

Rashid, M.M. 1999. SobgiBijnan. Rashid Publishing House. Dhaka, Bangladesh.

Reddy, K.N., Pattanaik, C., Reddy, C.S. and Raju, V.S. 2007. Traditional knowledgeon wild food plants in Andhra Pradesh. Indian J. Traditional Knowledge 6(1): 223-229.

Sarker, S.K. and Hossain, A.B.M.E. 2009. Pteridophytes of greater Mymensingh district of Bangladesh used as vegetables and medicines. Bangladesh J. Plant Taxon. 16(1): 47-56.

Saxena, R. 1999. How green is your diet? Nutrition 33(3): 9.

Su, Q., Rowley, K.G., Itsiopoulos, C. and O’Dea, K. 2002. Identification and quantitation of major carotenoids in select components of the Mediterranean diet: Green leafy vegetables, figs and olive oil. European J. Clin. Nutr. 56: 1149-1154.

Uddin, M.Z. and Hassan, M.A. 2014. Determination of informant consensus factor ethnomedicinal plants used in Kalenga forest, Bangladesh. Bangladesh J. Plant Taxon. 21(1): 83-91.

Uddin, M.Z., Kibria, M.G., and Hassan, M.A. 2015. Study of Ethnomedicinal Plants used by local people of Feni District, Bangladesh. J. Asiat. Soc. Bangladesh, Sci. 41(4): 735-757.

Vainio-Mattila, K., 2000. Wild vegetables use by the Sambaa in the UsambaraMountains, NE Tanzania. Ann. Bot. Fennici 37: 57-67. 
Vorster, I.H.J., Jansen van Rensburg, W., Van Zijl, J.J.B. and Venter, S.L. 2007. The importance of traditional leafy vegetables in South Africa. AfricanJournal of Food Agriculture Nutrition and Development 7(4), 1-13.

Woodcook, K.A. 1995. Indigenous knowledge and forest use: Two case studiesfrom the East Usambaras, Tanzania. East Usambaras Catchment For.Proj.Techn.Paper 22.For.and Beekeeping Div. and Finnish For. and ParkServ., Dar es Salaam and Vantaa. pp. 51.

Yusuf, M., Wahab, M.A., Choudhury, J.U. and Begum, J. 2006. Ethno-medico-botanical knowledge from Kaukhali proper and Betbunia of Rangamati district. Bangladesh J.Plant Taxon. 13(1): 55-61.

(Manuscript received on 14 May 2020; revised on 21 November 2020) 